\title{
Review series introduction
}

\section{Physiological systems under pressure}

\author{
Andrew R. Marks \\ Clyde and Helen Wu Center for Molecular Cardiology, Department of Physiology and Cellular Biophysics, and Department of Medicine, \\ Columbia University College of Physicians and Surgeons, New York, New York, USA.
}

\begin{abstract}
The marked disruption of the homeostasis of a physiological system, be it a cell, tissue, organ, or whole organism, is more commonly known as stress. In many ways, aging can be considered the ultimate stress. However, physiological systems are constantly exposed to more acute stresses. Advances in our understanding of the molecular response of several physiological systems to both physiologic and pathologic stress is discussed in this Review Series. It is hoped that such understanding will facilitate the development of approaches to ameliorate some of the limitations these stresses place on individuals. However, as pointed out in many of the articles, much remains to be learned before such approaches can be envisioned.
\end{abstract}

Fundamentally, all physiological systems are subject to, adapt to, are altered by, and ultimately succumb to distinct forms of stress. Stress can come in many forms, but for the purpose of this Review Series on molecular mechanisms of stress, it can be considered signals that substantially perturb the homeostasis of physiological systems. In a sense aging is the ultimate stress: the chronic and constant drip-drip-drip of time wears us all down. But what of more acute forms of stress, such as oxidative stresses, which wreak their effects at the cellular level, mechanical stresses, which put organs and tissues of the musculoskeletal system under pressure, and psychological stresses, which affect the whole organism? Can these forms of stress and their effects on the body be dealt with in a nontoxic, beneficial manner that ameliorates some of the limitations on our species, let alone the ravages of aging?

Much has been written in the social and psychology literatures about the impact of psychological stress on modern society. However, these polemics largely miss the point - even psychological stress is first and foremost a biological problem. Indeed, all physiological systems have well-defined limits within which they function quite well, but beyond which they fail. Defining these limits has been the work of centuries of biological study. With the advent of molecular biology and biophysics, it is now possible to begin to determine the molecular mechanisms by which physiological systems respond to stress and to explore how to expand, both pharmacologically and genetically, the physical and physiological limits of biological systems.

This Review Series on the molecular impact of different forms of stress on several physiological systems outlines recent advances in our understanding of how the environment bends physiological pathways and establishes their limitations. The topics addressed are merely examples of how certain physiological systems react to distinct forms of stress; they are by no means meant to be all-inclusive. As discussed in the articles in this series, although much has been learned recently about the molecular response of different physiological systems to stress, further work is required before we can overcome the limitations of these physiological systems and improve their response to stress. However, the practical applications and implications of improving the response of the physiological

Conflict of interest: The author is a consultant for Novartis; is the recipient of a research grant from Servier; owns shares in and chairs the scientific advisory board of ARMGO Pharma Inc., a start-up company that is developing ryanodine receptor-targeted therapy to improve exercise capacity; and is supported by a grant from DARPA. Citation for this article: J. Clin. Invest. 118:411-412 (2008). doi:10.1172/JCI34756. systems mentioned in this series - as well as others - to stress are vast and, if achieved, should improve the quality of life of humans. The ways in which new understanding about the impact of stress on physiological systems can be applied run the gamut from enabling individuals to more easily adapt to punishing environments (e.g., space travel), extreme climates on earth (e.g., high altitude and deserts), and increased population density (e.g., modern urban areas) to treating and/or preventing chronic diseases marked by physiological stress (e.g., heart failure) and ultimately to treating and/or preventing the stress of aging in the growing population of individuals in the last third of the human lifespan.

The first article in this Review Series, by Gregory Barton (1), discusses the response of innate immune cells to qualitatively distinct stresses in the form of tissue damage and infection by pathogenic and nonpathogenic microbes. Increasing evidence indicates that the germline-encoded receptors of the innate immune system that have evolved to recognize microbial products are also triggered by products of tissue damage. Barton suggests that the innate immune system distinguishes each of these different triggers of innate immune receptors and induces distinct inflammatory responses appropriate to the trigger.

The next 4 articles in the series address molecular mechanisms relevant to the response of tissues of the musculoskeletal system to physical and environmental stresses (2-5). Indeed, adaptation of the musculoskeletal system to stress remains one of the most important limitations to human physiology and affects us throughout life, from birth to old age. The physical and physiological limitations of our musculoskeletal system determine how fast and far we can run, how we adapt to harsh environments, how we recover from wounds and accidents, how we tolerate space travel in microgravity environments, and how we withstand the stress of aging as our musculoskeletal system weakens, bends, and breaks with alarming frequency.

A key clinical challenge in orthopedic and sports medicine is treating individuals whose tendons and ligaments have been overcome by physical stress (i.e., injured). In their Review, Dan Gazit and colleagues discuss the prospects for tendon regeneration (2). They address the challenges of regenerating tissues such as tendons and ligaments that are largely acellular structures composed of collagen and discuss the potential molecular targets that can be activated to produce new tendons. Growth factors serve as signals that lead to the generation and organization of new collagen fibers in healing tendons and, along with mechanical stimulation, determine the mechanical strength of regenerating tissue. Although it is hoped, as Gazit and colleagues note, that the advances in the 
molecular understanding of tendon morphogenesis and tendon neoformation will result in tendon regeneration strategies for use in the clinic, extensive studies, including analysis of therapeutic efficacy in multiple tendon injury models, are required before this can become a reality.

Bone fractures and osteoporosis are just some of the formidable clinical challenges posed by excessive physical stress on bone tissue. Currently, most drugs used for the prevention and treatment of osteoporosis block bone resorption, that is, they prevent further bone loss but do not promote bone formation. Recent advances have led to the introduction into the clinic of therapeutics that stimulate bone formation; these include recombinant bone morphogenetic proteins, which are FDA approved for spinal fusion and fracture healing, and parathyroid hormone, which is FDA approved for osteoporosis but can only be taken for 2 years in the United States. As Khosla and colleagues discuss, further improvements in the understanding of bone remodeling should enable the design of more effective approaches to manipulating the molecular pathways responsible for increasing bone formation in order to treat bone loss diseases and repair fractures (3). In this regard, one area of intense interest is furthering understanding of the cross-talk among the cells found in bone, which include bone-lining canopy cells, bone-lining cells on the bone surface, osteocytes, osteoblasts, and osteoclasts.

An overview of the impact of many types of stress, including physical and environmental stress, on the process of chondrogenesis (the dynamic cellular process that leads to the establishment of various types of cartilage) and chondrocyte differentiation is provided by Regis O'Keefe and colleagues (4). Many molecular regulators of chondrogenesis have been defined and are influenced by both normal and pathologic forms of stress. Of these, most is known about the effects on cartilage biology of stressors that arise from pathologic situations such as bone fractures, heterotopic ossification, and fibrodysplasia ossificans progressiva. However, as O'Keefe and colleagues note, a more detailed understanding of the effects on chondrogenesis and chondrocyte differentiation of normal mechanical stress will be necessary before therapies that facilitate cartilage repair can be envisioned.

The penultimate article in this Review Series, by Andrew Marks and colleagues, addresses the impact of physical stress on muscle function, in particular on the regulation of the calcium signals that control muscle contraction (5). Recently, the theory that lactic acidosis causes muscle fatigue has been called into question, opening the door for reexamination of the basic mechanisms underlying the limits to exercise capacity. Marks and colleagues focus on the potential role of dysregulation of sarcoplasmic reticulum (SR) calcium release induced by chronic physical stress, in the contexts of both chronic disease (e.g., heart failure) and strenuous exercise. How altered regulation of SR calcium release during stress might play a role in promoting the muscle damage that limits exercise capacity and how the study of genetic disorders suggests that leaky ryanodine receptor (RyR1) calcium release channels might cause skeletal muscle pathology are discussed. Based on these recent advances, the authors raise the possibility that novel drugs that can selectively fix the leak in RyR1 channels might protect against stress-induced muscle damage and improve exercise capacity under conditions of extreme physical stress.

One of the scourges of modern society is the widespread use of drugs to ameliorate the pressures of stress on the psyche and on human physiology at the whole-person level. In the final article in the series, Jessica Cleck and Julie Blendy examine the well-established connection between chronic psychological stress and drug addiction (6). They categorize drug addiction as a "chronic brain disease in which individuals cannot control their need for drugs, despite negative health and social consequences" (6). The premise is that the brain of an addicted individual responds to stress differently than does the brain of a nonaddicted individual. Cleck and Blendy focus their discussion on the common effects of stress and drugs of abuse on the brain, highlighting understanding gained from animal and human studies. These studies show that treatment of stress-related drug addiction can be a key to effective recovery. However, as they point out, although stress is a key factor linking reward to drug exposure and therapeutic drugs targeting the brain circuits linking stress and addiction are being examined for their treatment efficacy, further research examining withdrawal and relapse, in particular stress-induced relapse, is required to identify additional therapeutic targets.

Each of the articles in this Review Series addresses the impact of stress on a system that is critically important for survival and normal function. There are, however, many challenges ahead if we are to manipulate the response of an individual - at the cellular, tissue, organ, or even whole-body level - to stress. For example, it will be necessary to integrate new understandings of how stressinduced perturbations of homeostatic mechanisms contribute to disease and dysfunction in humans as well as in other species that are important for life on earth (witness the recent stress-induced collapse of bee colonies that threatened agriculture; ref. 7). Another challenge is how, based on these new understandings, to develop therapeutic strategies to maintain homeostasis in the face of toxic stresses and/or to return stress-perturbed systems to normal function. The challenges are enormous and will demand increased funding and innovative research approaches.

Address correspondence to: Andrew R. Marks, Clyde and Helen Wu Center for Molecular Cardiology, Department of Physiology and Cellular Biophysics, and Department of Medicine, Columbia University College of Physicians and Surgeons, New York, New York, USA. Phone: (212) 305-0270; Fax: (212) 305-3690; E-mail: arm42@columbia.edu.
1. Barton, G.M. 2008. A calculated response: control of inflammation by the innate immune system. J. Clin. Invest. 118:413-420.

2. Aslan, H., Kimelman-Bleich, N., Pelled, G., and Gazit, D. 2008. Molecular targets for tendon neoformation. J. Clin. Invest. 118:439-444.

3. Khosla, S., Westendorf, J.J., and Oursler, M.J. 2008. Building bone to reverse osteo- porosis and repair fractures. I. Clin. Invest. 118:421-428.

4. Zuscik, M.J., Hilton, M.J., Zhang, X., Chen, D., and O'Keefe, R.J. 2008. Regulation of chondrogenesis and chondrocyte differentiation by stress. J. Clin. Invest. 118:429-438.

5. Bellinger, A.M., Mongillo, M., and Marks, A.R. 2008. Stressed out: the skeletal muscle ryano- dine receptor as a target of stress. J. Clin. Invest. 118:445-453.

6. Cleck, J.N., and Blendy, J.A. 2008. Making a bad thing worse: adverse effects of stress on drug addiction. J. Clin. Invest. 118:454-461.

7. Cox-Foster, D.L., et al. 2007. A metagenomic survey of microbes in honey bee colony collapse disorder. Science. 318:283-287. 